\title{
Endoscopic removal of a migrated and long-indwelling self-expandable metal stent
}

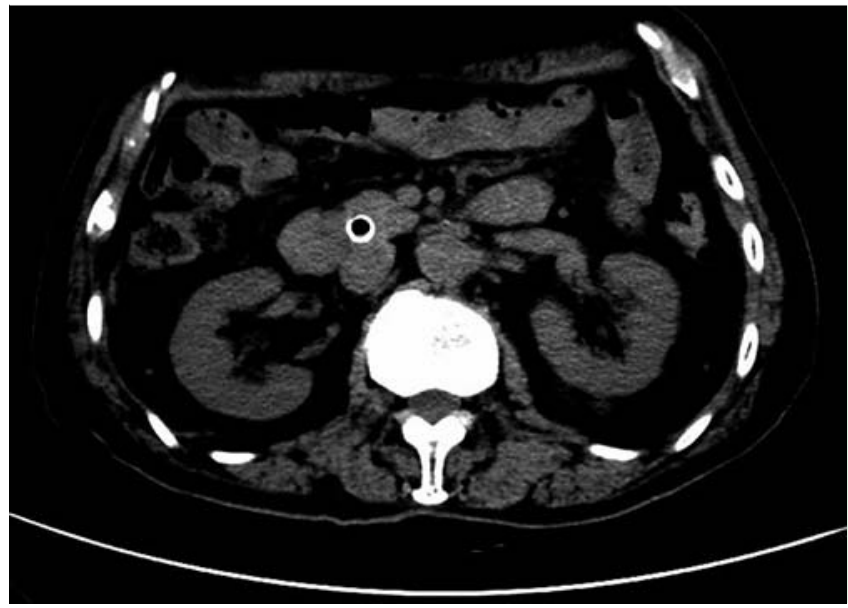

Fig. 1 Abdominal computed tomography demonstrates a hyperdense circular structure penetrating the duodenum. The uncovered self-expandable metal stent was previously placed for a biliary stricture that developed after the patient had undergone bile duct lithotomy.

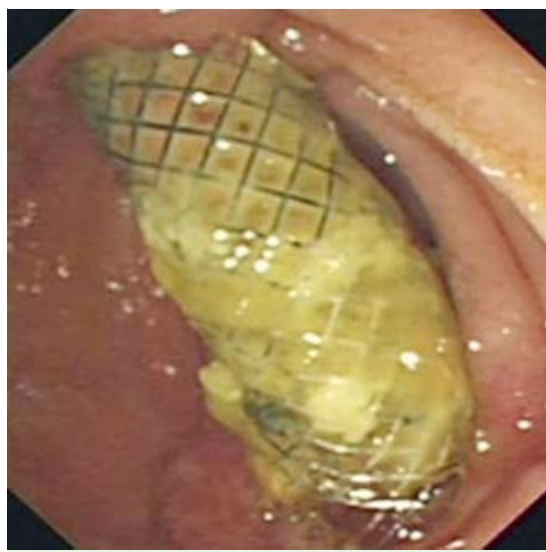

Fig. 2 The uncovered self-expandable metal stent has migrated distally and impacted the contralateral duodenal wall.

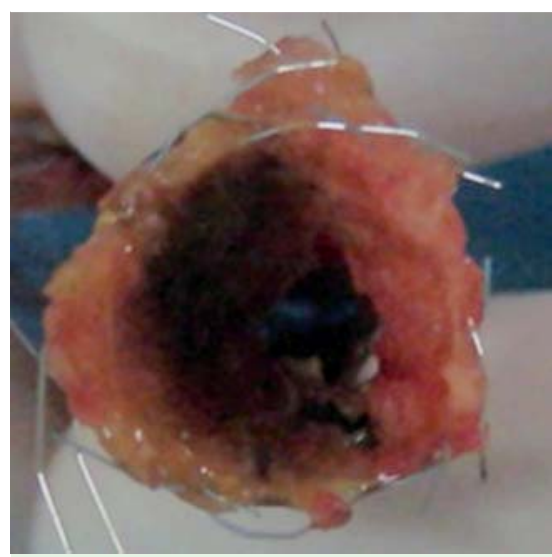

Fig.4 Frontal view of the uncovered selfexpandable metal stent.

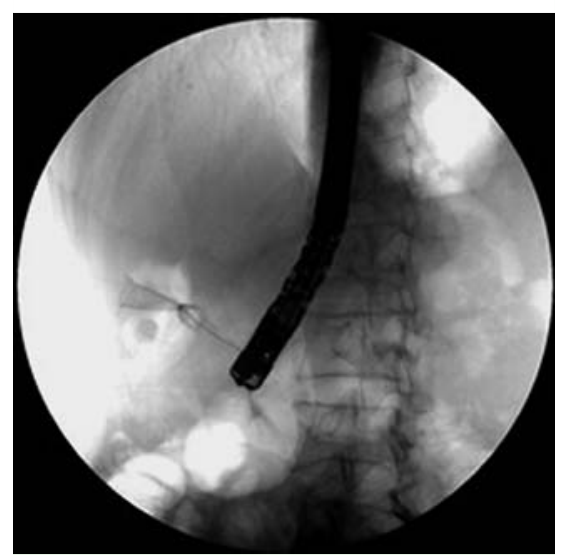

Fig. 3 Fluoroscopic image of stent removal with a snare.

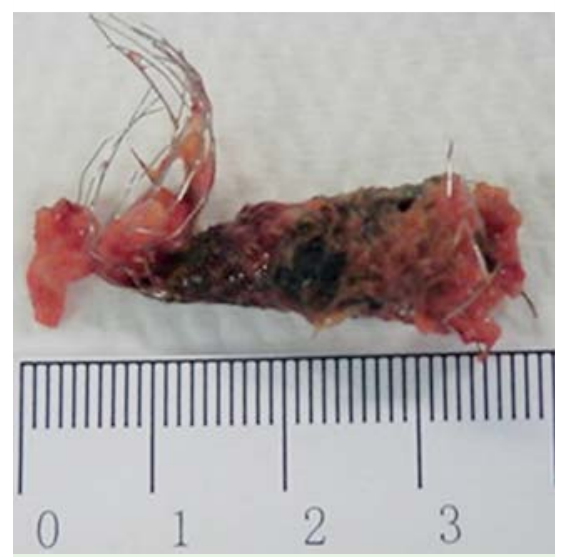

Fig. 5 Lateral view of the uncovered selfexpandable metal stent.
The removal of a biliary uncovered selfexpandable metal stent (uSEMS) is difficult or even impossible when the stent has been indwelling for longer than a couple of weeks because of tissue ingrowth $[1,2]$. A malfunctioning uSEMS may be removed with forceps, a snare, the invagination technique [3], or the stent-in-stent technique $[2,4]$. We report the removal of a migrated uSEMS, placed in a patient's bile duct 10 years earlier for a biliary stricture that had developed after bile duct lithotomy.

A 30-year-old man presented with abdominal pain and jaundice in 1984. Abdominal ultrasound revealed a large stone in the common bile duct (CBD). The patient underwent bile duct lithotomy and T-tube drainage. Approximately 18 years later, the patient was readmitted with a stenosis of the CBD, and a nitinol uSEMS (Micro-Tech, Nanjing, China) was placed. In 2012, the patient presented with abdominal pain and melena. His abdomen was soft. A slight tenderness of the upper quadrant with no rebound tenderness was noted. The complete blood cell count and blood chemistry were normal. Abdominal computed tomography revealed that the uSEMS had penetrated the duodenum ( $\bullet$ Fig. 1).

Because the total bilirubin and liver enzymes were normal, we thought that the epithelial hyperplasia might not be diffuse or severe and that the stent might be removed with a snare [1]. The uSEMS protruded into the duodenal lumen and impacted the contralateral duodenal wall

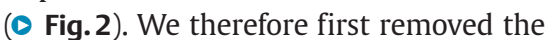
exposed wire ends of the stent with biopsy forceps; clockwise torsion and a backand-forth movement were then applied. Finally, the uSEMS was successfully removed with a snare ( $\bullet$ Figs. $3-5$ )

Thus far, the patient remains well. Additionally, there is no evidence of malignancy or a need for biliary stenting.

Endoscopy_UCTN_Code_TTT_1AR_2AZ

Competing interests: None

\section{Qi-Shan Zeng, Hong-Lin Chen, Qin Liu, Hong-Ze Zeng, Qi-Ming Wang, Wei Liu, Bing $\mathbf{H u}$}

Department of Gastroenterology, West China Hospital, Sichuan University, Chengdu, Sichuan, China 


\section{References}

1 Familiari P, Bulajic M, Mutignani $M$ et al. Endoscopic removal of malfunctioning biliary self-expandable metallic stents. Gastrointest Endosc 2005; 62: 903 -910

2 Arias Dachary FJ, Chioccioli C, Deprez PH. Application of the "covered-stent-in-uncovered-stent" technique for easy and safe removal of embedded biliary uncovered SEMS with tissue ingrowth. Endoscopy 2010; 42: E304-E305

3 Nakai Y, Isayama $\mathrm{H}$, Kawakubo K et al. Endoscopic removal of a biliary covered metallic stent with the invagination method. Endoscopy 2011; 43: E30-E31
4 Tan DM, Lillemoe KD, Fogel EL et al. A new technique for endoscopic removal of uncovered biliary self-expandable metal stents: stent-in-stent technique with a fully covered biliary stent. Gastrointest Endosc 2012; 75: 923-925

\section{Bibliography}

DOI http://dx.doi.org/

10.1055/s-0034-1392207

Endoscopy 2015; 47: E299-E300

(c) Georg Thieme Verlag KG

Stuttgart · New York

ISSN 0013-726X
Corresponding author

\section{Bing Hu, MD}

Department of Gastroenterology West China Hospital

Sichuan University

No. 37 Guo Xue Xiang

Chengdu

Sichuan, 610041

People's Republic of China

Fax: +86-28-85501670

hubingnj@163.com 